\title{
BMJ Open Bibliometric analysis of gaps in research on asbestos-related diseases: declining emphasis on public health over 26 years
}

\author{
Ro-Ting Lin, ${ }^{1,2}$ Matthew John Soeberg, ${ }^{2}$ Lung-Chang Chien, ${ }^{3}$ Scott Fisher, ${ }^{4}$ \\ Jukka Takala, ${ }^{5}$ Richard Lemen, ${ }^{6}$ Tim Driscoll, ${ }^{7}$ Ken Takahashi ${ }^{2}$
}

To cite: Lin R-T, Soeberg MJ, Chien L-C, et al. Bibliometric analysis of gaps in research on asbestos-related diseases: declining emphasis on public health over 26 years. BMJ Open 2018;8:e022806. doi:10.1136/ bmjopen-2018-022806

- Prepublication history and additional material for this paper are available online. To view these files, please visit the journal online (http://dx.doi. org/10.1136/bmjopen-2018022806).

Received 7 March 2018 Revised 14 May 2018

Accepted 8 June 2018
Check for updates

(C) Author(s) (or their employer(s)) 2018. Re-use permitted under CC BY-NC. No commercial re-use. See rights and permissions. Published by BMJ.

For numbered affiliations see end of article.

Correspondence to

Dr Ken Takahashi;

ken.takahashi@sydney.edu.au

\section{ABSTRACT}

Objectives The global burden of asbestos-related diseases (ARDs) is significant, and most of the world's population live in countries where asbestos use continues. We examined the gaps between ARD research and suggestions of WHO and the International Labour Organization on prevention.

Methods From the Web of Science, we collected data on all articles published during 1991-2016 and identified a subset of ARD-related articles. We classified articles into three research areas-laboratory, clinical and public health-and examined their time trends. For all and the top 11 countries publishing ARD-related articles, we calculated the proportions of all ARD-related articles that were in each of the three areas, the average rates of ARDrelated articles over all articles, and the average annual per cent changes of rates.

Results ARD-related articles $(n=14284)$ accounted for $1.3 \%$ of all articles in 1991 , but this had declined to $0.8 \%$ o by 2016 . Among the three research areas, the clinical area accounted for the largest proportion (65.0\%), followed by laboratory (26.5\%) and public health (24.9\%). The public health area declined faster than the other areas, at $-5.7 \%$ per year. Discrepancies were also observed among the top 11 countries regarding emphasis on public health research, with Finland and Italy having higher, and China and the Netherlands lower, emphases.

Conclusions There is declining emphasis on the public health area in the ARD-related literature. Under the ongoing global situation of ARD, primary prevention will remain key for some time, warranting efforts to rectify the current trend in ARD-related research.

\section{INTRODUCTION}

WHO and the International Labour Organization (ILO) have repeatedly stated that the best way to eliminate asbestos-related diseases (ARDs) is for countries to stop using asbestos. ${ }^{1-3}$ Although more than 60 countries have banned partial or all use of asbestos, ${ }^{4}$ the majority of the world's population currently live in countries with ongoing asbestos consumption. ${ }^{5}$ In 2015 , asbestos was mined in five countries, while in 2014 at least 30 countries imported raw asbestos, and presumably a much larger number of countries consumed

\section{Strengths and limitations of this study}

The main strength of the study is that it contributes to the estimation of relative gaps of research focus in asbestos and asbestos-related diseases globally using a longitudinal analysis.

- We comprehensively collected data on the number of articles published during 1991-2016, classified 252 subject categories into three research areas, and assigned each article into these research areas, respectively.

- The 26-year data showed the number of articles increased over the last quarter century, but the expansion has lagged behind that of the entire scientific literature.

- Although the data were extracted for all countries with available data, countries that have limited publications might be under-represented.

- Other asbestos-related diseases might be under-represented in our analysis due to our selection criteria for articles.

asbestos-containing products. ${ }^{6}$ A recent Global Burden of Diseases (GBDs) study estimated that in 2016 more than 222000 deaths were attributable to occupational exposure to asbestos. ${ }^{7}$ ARDs, including asbestos-related cancers such as mesothelioma and lung cancer, ${ }^{8}$ are caused by occupational as well as environmental exposure to asbestos. ${ }^{2}$ The incidence rates of ARD are expected to peak in the coming decades as a consequence of past and ongoing exposure to asbestos as well as the long latency period between exposure and disease development. ${ }^{2} 9$ Odgerel et al recently have shown that the GBD estimates may still be significantly underestimated. ${ }^{8}$

Tackling the burden of ARD requires policies based on sound scientific knowledge. ${ }^{10}$ Relevant and high-quality research on asbestos and ARDs should provide the evidence base required for the prevention of asbestos exposure, clinical intervention, patient care and policy-making. ${ }^{511} 12$ Although the scientific community has long been engaged in 
research on a wide spectrum of themes related to asbestos and ARDs, resulting in many publications (ARD-related articles, hereinafter), the extent to which such research aligns with societal priorities reflected in the WHO/ILO statements is unclear. Moreover, variations in the topics addressed in ARD-related articles in relevant research among countries and over time is a separate, but related theme of interest. Bibliometric analysis of the global ARD-related literature could shed light on the quantity (or 'number') and the quality (or 'articles published in a specific research area'). ${ }^{13}$ The results of such an analysis may also assist in optimising resource allocation and the translation of research into policy.

The ongoing global situation in relation to ARDs and the WHO/ILO statements lend support to the notion that public health and disease prevention should be areas of high priority in research on ARDs. ${ }^{1-3}$ Although the overall trends in the mesothelioma literature have been analysed in previous studies, ${ }^{14}{ }^{15}$ to date no analysis of the ARD-related literature has been conducted aimed at elucidating the research priorities in previous works while accounting for the different areas of scientific expertise. Therefore, the objectives of this study were to describe trends in ARD-related research with an emphasis on the area of public health and intercountry variations by analysing the numbers and research areas of articles published during 1991-2016.

\section{METHODS}

\section{Data source}

We searched for ARD-related publications in the database 'Web of Science Core Collection' on the Web of Science platform (Clarivate Analytics) by using the keywords 'asbestos' OR 'mesothelioma'. We entered our keywords under the search field 'Topic', which includes Title, Abstract, Author Keywords and Keywords Plus on the Web of Science platform. ${ }^{16}$ The inclusion criteria were original scientific articles or reviews (articles, hereinafter) and year of publication during 1991-2016. We chose this period for two reasons. First, the International Classification of Diseases 10th version (ICD-10), which assigned the code C45 to 'malignant mesothelioma', was endorsed by the 43rd World Health Assembly in 1990. Second, the yearly number of ARD-related articles increased markedly after 1991. ${ }^{17}$ Data obtained from the Web of Science were imported into the InCites (Clarivate Analytics) tool for further grouping and analyses. ${ }^{17}$

\section{Group definition and process}

To assess the trends of ARD-related research in specific research fields, we classified the Web of Science's 252 subject categories into three areas: ${ }^{18}$ (1) laboratory: including toxicology, cell biology, biochemistry and molecular biology, etc; (2) clinical: including oncology, respiratory system, pathology, etc; (3) public health: including public, environmental and occupational health, environmental sciences, environmental engineering, etc and (4) irrelevant: not $(1,2)$ or $(3) .{ }^{18}$ There are three premises to assign each article to relevant subject categories. First, each article was published in only one journal. Second, each journal, according to the design of the Web of Science platform, can be assigned to one or more relevant subject categories. ${ }^{18}$ Third, each category, according to our grouping approach, can be assigned to only one research area. Hence, according to above three premises, each article was assigned by the Web of Science to a single or multiple subject categories, but each category was classified to only one research area. This allowed us to use the Web of Science's 252 subject categories as surrogate indicators of the research focus of each article. ${ }^{18} \mathrm{We}$ assigned these subject categories to the three research areas via a three-step process. First, two researchers independently classified the 252 subject categories into the three areas with $72 \%(\mathrm{n}=182)$ agreement. Second, a senior rater independently assigned the categories to the three areas with no knowledge of the assignments of the two researchers for the disputed cases $(n=70)$ and then reached $96 \%$ agreement $(\mathrm{n}=243)$. Finally, for the remaining $4 \% \quad(n=9)$ of subject categories without agreement, the three researchers discussed each subject category and reached a consensus on the area to which it should be assigned. Articles in research areas $(1,2)$ and (3) were extracted for further analyses. The assignments of the subject categories to the three areas are listed in online supplementary file, table S1, including laboratory $(n=22)$, clinical $(n=38)$ and public health $(n=32)$.

\section{Analysis}

We calculated the numbers and proportions of ARD-related articles in each research area for all countries and for the top 11 countries. These 11 countries accounted for 83.0\% of ARD-related articles during 1991-2016. We treated all articles in research areas $(1,2)$ and (3) in InCites as a reference group.

To estimate the linear trend of ARD-related articles over time, we applied the joinpoint regression modelling approach to consider the years with significant joinpoints (the maximum number of nodes was two in each model) and calculated the average annual per cent change (AAPC, a weighted average of yearly change), ${ }^{19}$ by fitting a log-linear regression:

$$
\ln (\text { publication rate })=\beta_{0}+\beta_{1} \times(t)
$$

where $\mathrm{t}$ denotes the calendar time, from 1 for the year of 1991 to 26 for the year of 2016 , and $\beta_{0}$ and $\beta_{1}$ denote the intercept and slope. We further applied a generalised additive mixed model to examine the intercountry variations in the rate of ARD-related articles relative to all articles. ${ }^{20}$ We adjusted for per capita asbestos consumption (defined as the volume of asbestos production and importation divided by the total population in each year) ${ }^{21} 22$ and the age-adjusted mortality rate of mesothelioma (calculated by dividing the number of mesothelioma deaths (C45 in ICD-10) of 5-year age groups by the population of corresponding age groups in each 
year during 1995-2013, weighted by the world standard population). ${ }^{8} Y_{i t}$ was defined as the number of ARD-related articles in county $i$ at calendar time $t$, which follows a Poisson distribution with a mean parameter $\mu_{i t}$. The model equation can be expressed as follows:

$$
\ln \left(\mu_{i t}\right)=\alpha+\alpha_{i}+f(t)+X_{i t}^{A S B}+X_{i t}^{M O R}+\text { offset }
$$

where $\alpha$ and $\alpha_{i}$ denote the fixed and random intercepts. $X_{i t}^{A S B}$ and $X_{i t}^{M O R}$ represent asbestos consumption and the age-adjusted rate of mortality from mesothelioma in country i at time t. A cubic spline $f(t)$ was included to control for temporal autocorrelation. Offset denotes the natural logarithm of all articles in each country per year. The final model for each research area only included covariates that were significantly associated with ARD-related articles. We performed the statistical analyses using Joinpoint Regression Program V.4.5.0.1 (National Cancer Institute, USA) and R studio V.1.0.143 (R Foundation for Statistical Computing, Austria). The significance level was set at $5 \%{ }^{23}$

\section{Patient and public involvement}

No patients and or public were involved in this study.

\section{RESULTS}

We identified 15405 ARD-related articles published during 1991-2016 in the Web of Science, of which 14284 had information regarding the year of publication, the country of authors and the subject categories to analyse using InCites for categorisation into the three research areas (see online supplementary file, table S1).

As shown in figure 1, both the total number of scientific articles and the number of ARD-related articles increased during 1991-2016, but the proportions of all articles that were ARD-related decreased, from 1.3\%o (proportion multiplied by 1000) in 1991 to $0.8 \%$ o in 2016 .

Figure 2 shows the trends in the proportions of articles that were in each of the three research areas among all and ARD-related articles during 1991-2016. Note that, in each year, the sum of the proportions of the three areas exceeded $100 \%$ because each article could be classified into more than one area. Among all articles, the proportion in the public health area grew over the study period to narrow the gap with the other two other areas (figure 2A). In contrast, for ARD-related articles, the proportion in the public health area fluctuated but steadily decreased (from $39.3 \%$ in 1991 to $22.5 \%$ in 2016), whereas the proportion in the clinical area fluctuated but generally increased and that in the laboratory area fluctuated with no discernible trend (figure 2B).

Among all ARD-related articles published in all countries during 1991-2016, the clinical area accounted for the largest proportion $(65.0 \%)$, followed by the laboratory $(26.5 \%)$ and public health $(24.9 \%)$ areas (table 1$)$. In terms of the rate of ARD-related articles relative to all articles, the clinical area was the highest (1.3\%o/year), followed by the public health $(0.8 \%$ / year) and laboratory $(0.6 \%$ o/year) areas. The AAPC of ARD-related articles relative to all articles was negative with no statistical significance for the clinical area $(-0.1(95 \%$ CI -0.5 to 0.4$) \% /$ year), and negative with statistical significance for the public health $(-5.7$ (95\% CI -7.6 to -3.8$) \% /$ year $)$ and laboratory $(-1.1(95 \%$ CI -2.0 to -0.3$) \% /$ year $)$ areas.

When ARD-related articles from the top 11 countries were analysed, similar patterns were observed to those found for all countries. However, distinctive patterns emerged when the 11 top countries were examined individually. In terms of relative proportions, the clinical area was dominant in all 11 countries, with Australia showing

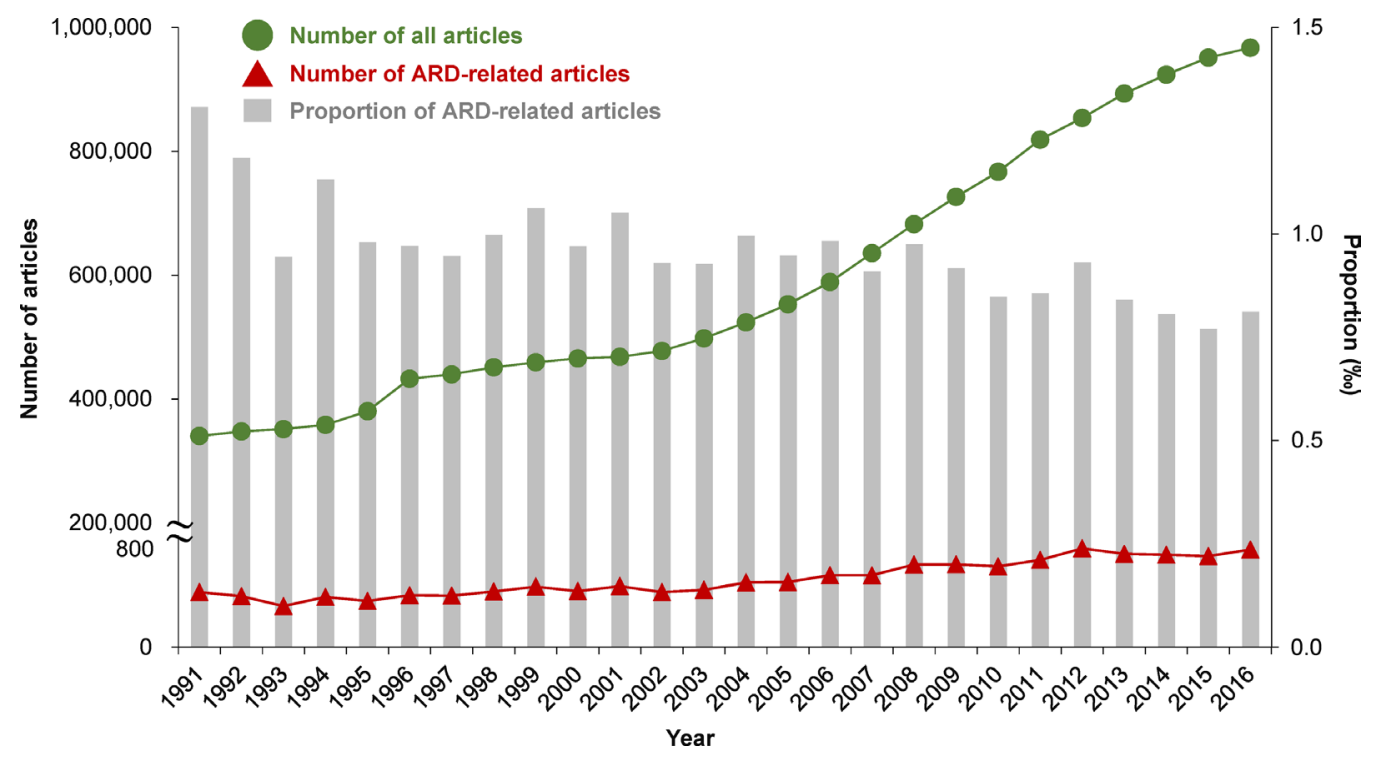

Figure 1 Trend in the number and proportion of scientific articles from 1991 to 2016. ARD-related articles=articles with a theme of asbestos and ARDs. Articles were defined as articles or reviews belonging to any of the three research areas (see online supplementary file, table S1) in InCites (Clarivate Analytics). ${ }^{17}$ ARD, asbestos-related diseases. 
(A) All publications

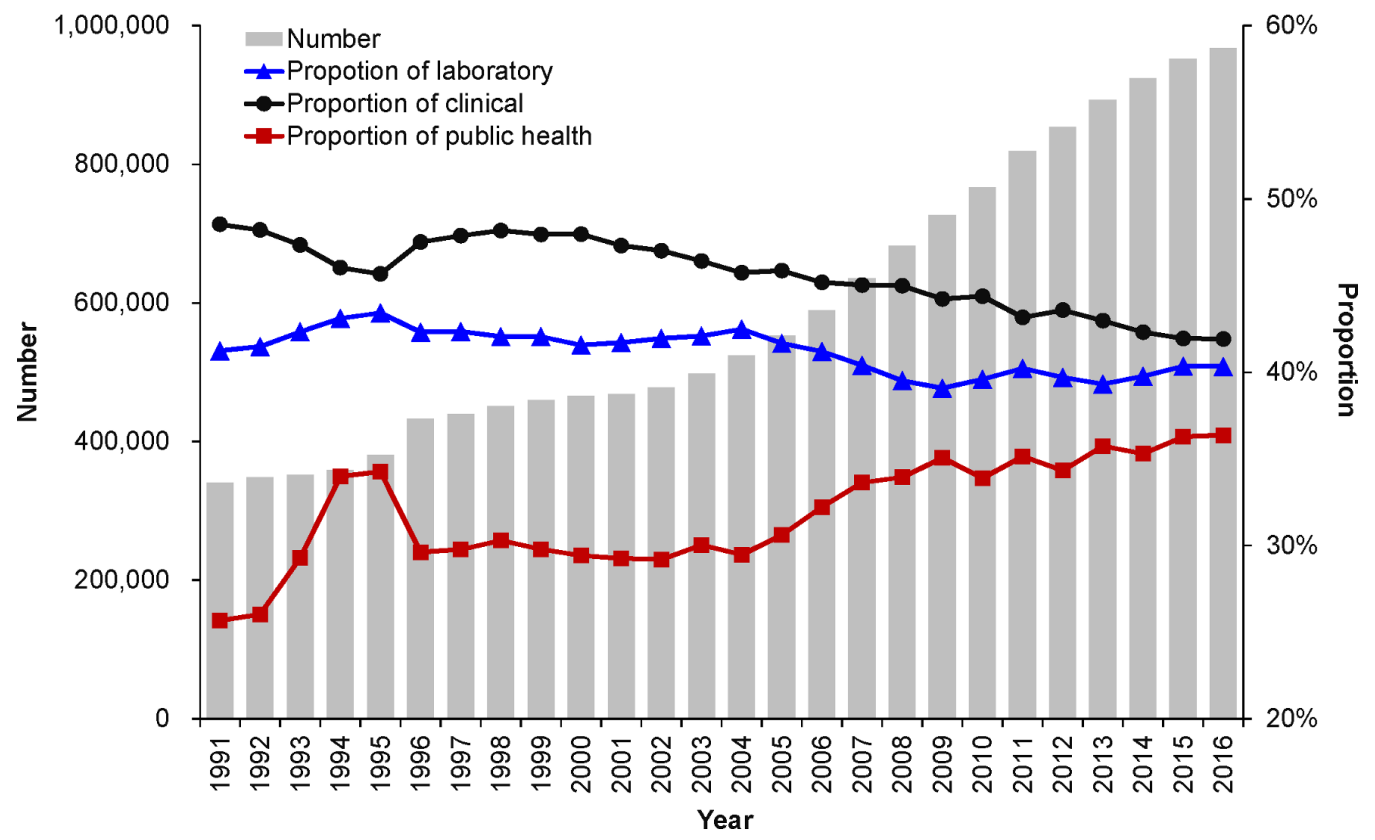

(B) ARD-related publications

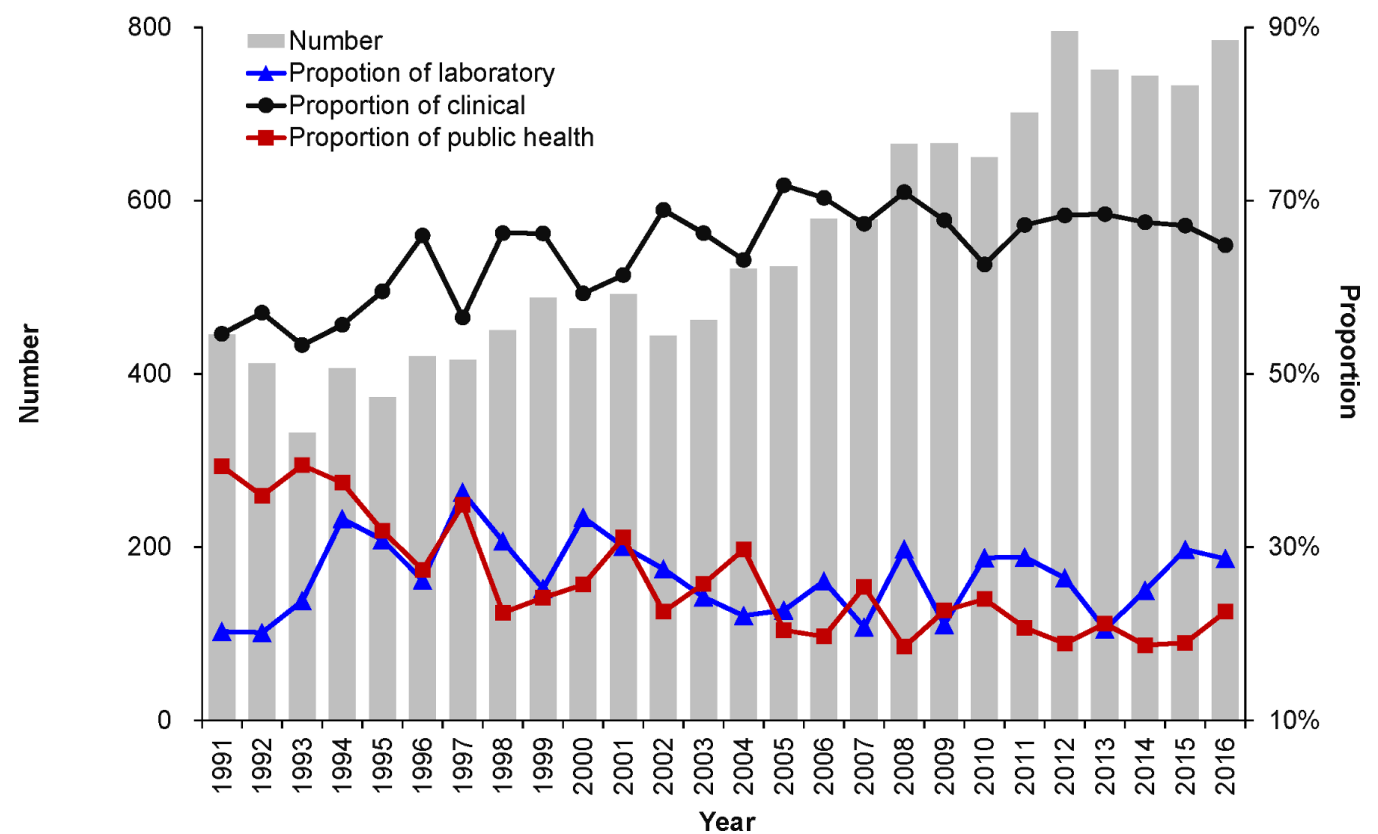

Figure 2 Trend in the number and proportion of articles by research area. ARD-related publications=articles with a theme of asbestos and ARDs. Articles were defined as articles or reviews belonging to any of three research areas (see online supplementary file, table S1) in InCites (Clarivate Analytics). ${ }^{17}$ ARD, asbestos-related diseases.

the highest proportion $(75.0 \%)$ and Canada the lowest $(56.2 \%)$. When the areas of public health and laboratory were compared, seven countries had higher proportions for laboratory and four countries had higher proportions for public health. In terms of rate, 9 out of the 11 countries had the highest rates of ARD-related articles for the clinical area among the three areas, led by Australia (2.2\%o/year). In the public health area, Finland and Italy had the highest rates of ARD-related articles (4.2\%o/ year and $2.8 \%$ /year, respectively), while the Netherlands and China had the lowest $(0.5 \%$ /year and $0.6 \%$ /year, respectively).

In terms of the AAPC of ARD-related relative to all articles among the top 11 countries (see online supplementary file, figure S1), the clinical area showed statistically significant increases in two countries, specifically, Japan (4.3 (95\% CI 0.5 to 8.3$) \% /$ year) and Italy (2.6 (95\% CI 1.6 to 3.6$) \%$ /year), but statistically significant decreases in five countries. Similarly, the laboratory area showed statistically significant increases in Japan $(5.9$ (95\% CI 


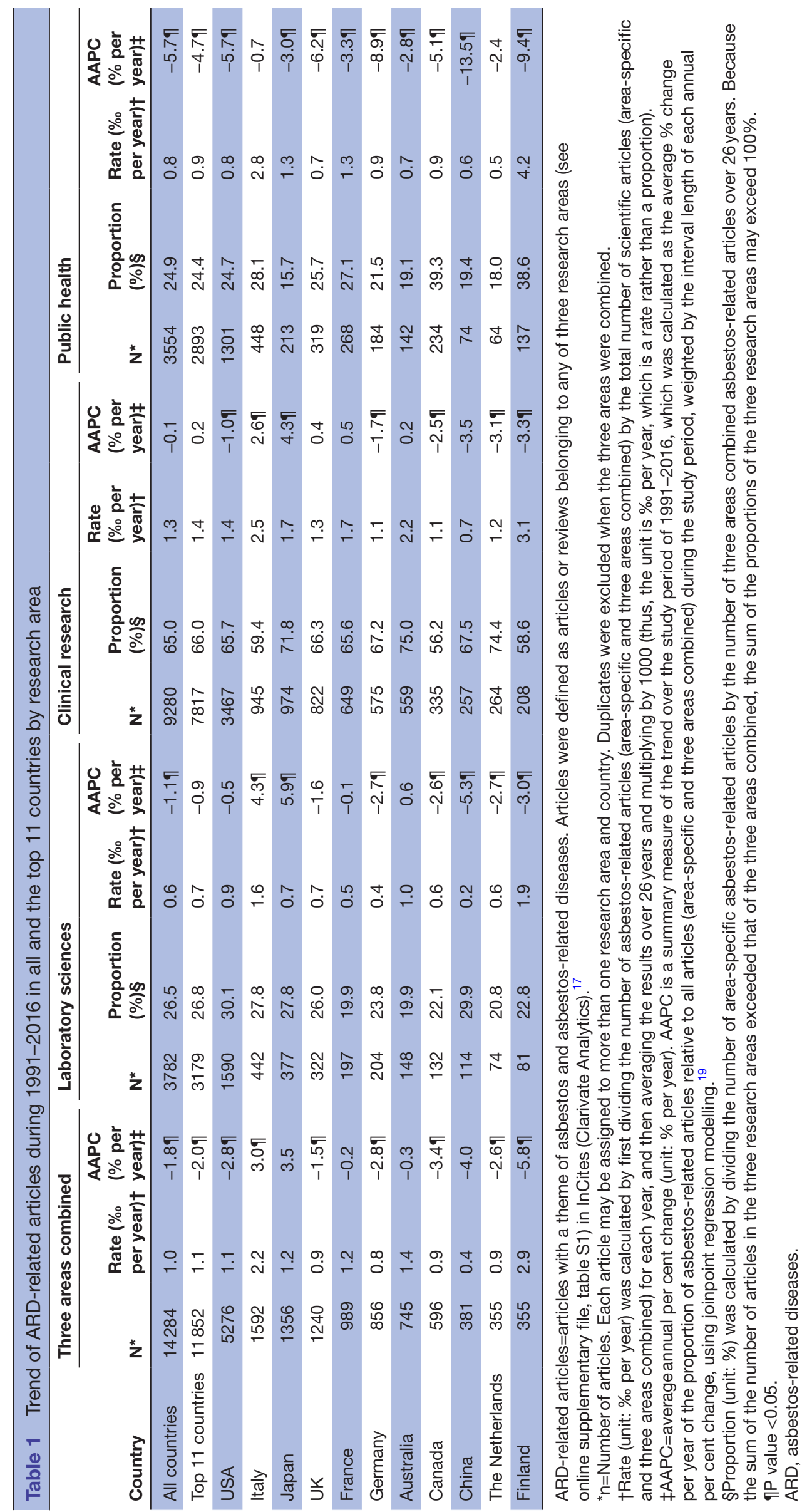




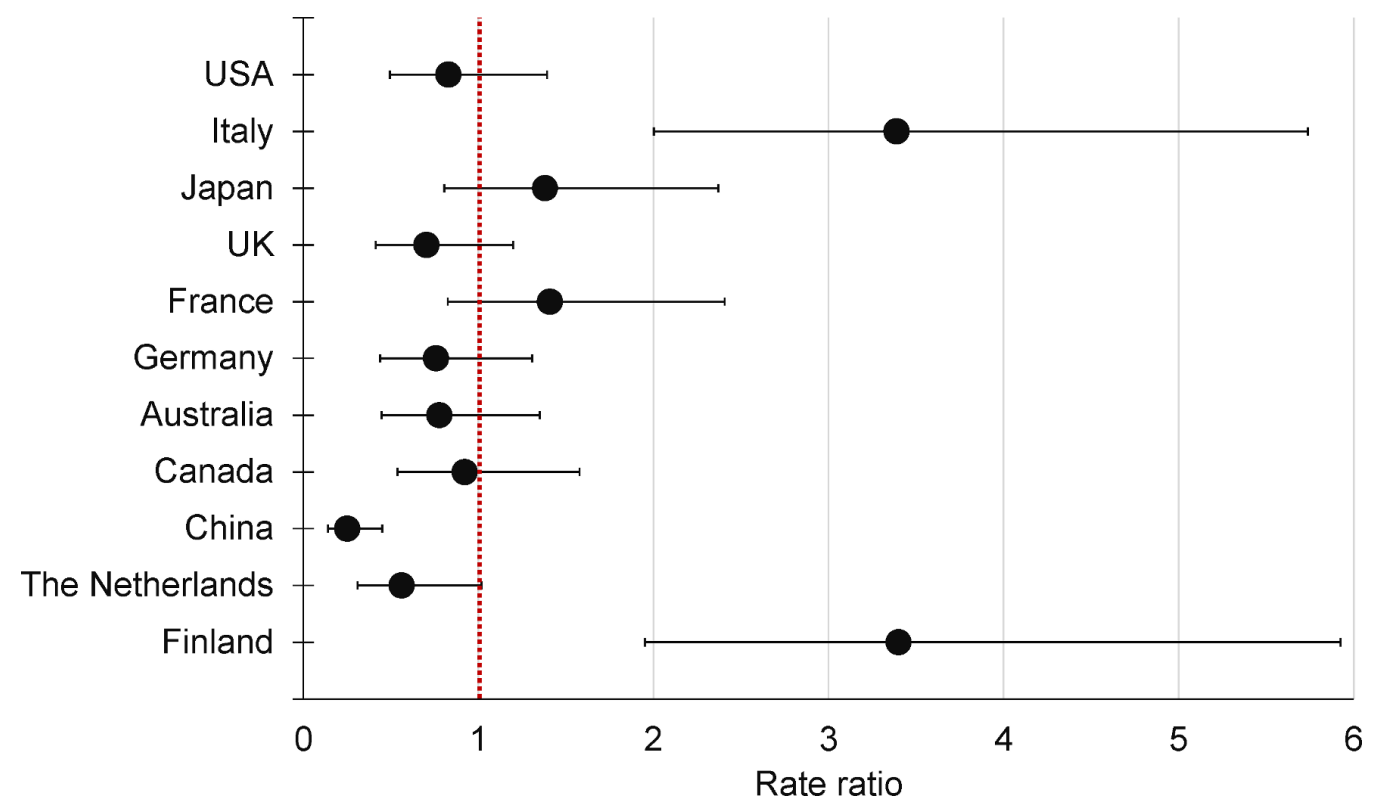

Figure 3 Rate ratio of ARD-related articles in the public health area in each of the top 11 publishing countries compared with the average for the top 11 countries ARD-related articles=articles with a theme of asbestos and ARDs. Red dotted line represents the average of the top 11 countries. The rate ratio was estimated using a generalised additive mixed model with a Poisson distribution by comparing the rate of the public health area of ARD-related articles of each country to the average rate of the public health area of asbestos-related articles in these 11 countries (rate ratio=1), after adjusting for temporal variation. Other covariates, specifically asbestos consumption and age-adjusted mortality rate of mesothelioma, were not included in the final model because they did not reach statistical significance. ARD, asbestos-related disease.

3.4 to 8.4$) \%$ /year) and Italy (4.3 (95\% CI 2.9 to 5.7$) \% /$ year), but statistically significant decreases in five countries. The public health area decreased in all 11 countries, with statistical significance in nine countries, led by China ( -13.5 (95\% CI -16.2 to 10.7$) \%$ /year).

Figure 3 compares the top 11 countries in terms of the rate ratio of the public health area in ARD-related articles, after adjusting for temporal variation. (Note that asbestos consumption and age-adjusted mortality rate of mesothelioma were not statistically significant predictors of an article being in the public health area, and were thus excluded from the final model.) Compared with the average rate across the 11 countries, Finland and Italy showed significantly higher rate ratios by 3.40 -fold (95\% CI 1.95 to 5.92) and 3.39-fold (95\% CI 2.00 to 5.74), respectively. In contrast, China showed a significantly lower rate ratio by 0.25 -fold ( $95 \%$ CI 0.14 to 0.45$)$. The Netherlands had the second lowest rate ratio, but it did not reach statistical significance at 0.56 -fold (95\% CI 0.31 to 1.02$)$.

\section{DISCUSSION}

Our analysis of trends in scientific articles published throughout the world between 1991 and 2016 revealed that the growth of ARD-related articles published lagged considerably behind that of the overall scientific output. We further examined the proportions of papers published in three research areas of interest-public health, laboratory and clinical-both in the entire and ARD-related literature. We found that, in the entire literature, the proportion of papers with a public health orientation was initially much lower than those of laboratory and clinical, but that during the study period the proportion of public health-oriented papers grew, narrowing the gap among the three areas. When we analysed only the ARD-related literature, by contrast, the public health area did not keep up with the clinical area and criss-crossed with the laboratory area. Although the clinical area was consistently dominant, individual countries showed distinct patterns in the proportions of articles in the three research areas and the trends in those proportions over time. Overall, the presence of ARD-related articles has been weakening in the scientific literature, which was enhanced by a decline in emphasis on public health in ARD-related research. This raises serious concerns in view of the ongoing relevant global situation and the WHO/ILO declarations. ${ }^{23}$

ARD is increasingly recognised as a global health issue, where a variety of unmet needs require multidisciplinary cooperation on a global scale. ${ }^{59} \mathrm{WHO}$ has been joined by the ILO to emphasise that implementing public health measures is the most effective approach to eliminating ARD. ${ }^{2324}$ This is plausible as the majority of ARD cases are reported in developed countries that already have an asbestos ban, but an estimated 125 million people, primarily in low/middle-income countries, continue to be occupationally exposed to asbestos. ${ }^{2}$ These countries typically lack the medico-social infrastructure to prevent asbestos exposure and to deal adequately with patients, including diagnosis, treatment, care and compensation. The relevant experience and expertise acquired by 
developed countries have yet to be shared with the countries that are in need. Our findings in regard to the trends in ARD-related research, specifically the slow growth of ARD-related research in general and a lack of emphasis on the public health area in particular, indicate a failure to support the global health cause and the WHO/ILO positions.

Our analysis showed that ARD-related research has recently been gravitating towards the clinical area. This is corroborated by an earlier study which found an association between ARD-related research output and national burden of mesothelioma, although that study did not differentiate between research areas. ${ }^{15}$ We found that countries with higher age-adjusted rates of mortality from mesothelioma had higher numbers of ARD-related articles, but only for the clinical area and the three areas combined (data not shown). In addition, our selection criteria of ARD-related articles (ie, 'asbestos' OR 'mesothelioma') likely increased the proportion of articles with a clinical emphasis. A preponderance of clinical papers may be explained by the important themes explored in clinical ARD-related research, such as methods for improving the diagnosis and treatment of patients. Moreover, the proportion of clinical papers may be inflated by many papers in the laboratory area having a clinical aspect, but not vice versa. That is, ARD-related laboratory research addressing important themes, such as improving our understanding of the disease mechanism, may be conducted in the context of clinical and/or public health research. Moreover, the laboratory area is probably driven in tandem with the clinical area by the growing emphasis on translational research in which laboratory work is positioned as 'preclinical.'

A key factor that may support or impede the growth of ARD-related research in the public health area is funding due to its known substantial impact on the quantity and quality of scientific articles. For example, austerity policies on public health in general can also impact public health research. Funding agencies usually allocate a larger portion of funding to emerging challenges or high priority research programmes that are in line with their policy direction. ${ }^{25}$ The majority of research funding comes from governments, ${ }^{26}$ but diverse funding structures among countries lead to different research agendas in public health. ${ }^{27}$ For example, in most countries in the European Union, the Ministry of Science or Ministry of Education is the primary funding agency for public health research. ${ }^{27}$ Funding of medical research can cover the public health area, but public health usually accounts for only a small portion of the entire medical research budget. ${ }^{27}$ The lack of national public health research strategies when allocating funds or unsustainable investment in public health research could downplay the importance of public health science. ${ }^{28}$ Furthermore, the capacity of national insurance system to detect and deal with ARD is an important factor, ${ }^{29-31}$ whereby a limited capacity will obstruct visibility in the public arena, the public agenda and hence public funding. Further research is warranted to assess the funding situations of ARD-related research and how they relate to research areas.

Although the rate of articles published in the ARD-related literature in the public heath area consistently declined across the top 11 countries, Finland and Italy maintained a greater emphasis on public health research, whereas China and the Netherlands had the least emphasis. We speculate that the following factors may have been in play. Previous studies have highlighted that Nordic countries had more publications in occupational and environmental health compared with other European countries. ${ }^{32}$ Finland has been the dominant country in occupational and environmental health research (accounting for $28 \%$ of public health research in Finland vis-à-vis $11 \%$ in the Netherlands and $8 \%$ in the UK) ${ }^{32}$ In addition, the Finnish Institute of Occupational Health implemented many initiatives to address the global asbestos/ARD situation, including international conferences that formulated the Helsinki Criteria. ${ }^{33} 34$ In Italy, since the banning of asbestos in 1992, nationwide recognition grew regarding the need to initiate a permanent surveillance system for mesothelioma, which culminated in the establishment of the National Register of Malignant Mesothelioma in 2002. ${ }^{35}$ This had the additional effect of promoting research, ${ }^{35}$ most likely in the public health area. In China, researchers may be less incentivised to conduct ARD-related research, particularly on the public health aspect, as it is a country where the production and consumption of asbestos remain rampant, with government oversight. The Netherlands accelerated translational research in medicine (ie, from bench to bedside), to become the central hub of European Advanced Translational Research Infrastructure in Medicine integrating basic and clinical research. This may have shifted emphasis away from public health to the laboratory and clinical areas.

The present study had several limitations. First, we classified research into three areas based on the subject categories provided by the Web of Science, ${ }^{18}$ which may have led to misclassifications. For example, some laboratory studies may have been conducted in the context of public health research, but our method was unable to discern context. Each article may also be assigned to more than one country because the article was contributed by authors from different countries. However, any misclassification bias will have been reduced by the capacity of our method to assign each article to multiple categories and to multiple countries. An inevitable side effect, however, was that the sum of the number of articles in the three research areas exceeded that of the three areas combined. Similarly, the sum of the number of articles for each country exceeded that of all countries. Second, when making longitudinal comparisons, we focused on countries that have published higher numbers of ARD-related articles. However, the ARD burden will eventually shift to low/middle-income countries that currently use asbestos. In those countries, low numbers of ARD-related articles were published during the study period, which 
limited the information to be analysed. Third, some ARDs, such as asbestos-related lung cancer, would have been under-represented in our analysis due to our selection criteria for articles. However, the inclusion of lung cancer, as it relates to a wide range of causal factors other than asbestos, would have reduced the specificity for ARD-related articles. We believe that our criteria enabled a reasonable representation of ARD-related articles.

For society to effectively tackle a research theme such as ARD, research efforts and resources must be apportioned according to the priority needs under conditions of limited resources. To achieve this, research trends should be constantly reviewed to determine whether those needs are being addressed, and if not, to adjust the focus of effort. Developed countries are contending with a lingering ARD epidemic as well as a long-term issue of exposure to in situ asbestos. Moreover, various low/ middle-income countries are faced with an emerging ARD epidemic compounded by ongoing exposure to asbestos in current use. Clearly, from the standpoint of ARD prevention, the public health area of research needs strengthening. In doing so, attention should be extended to the many subareas of public health, including health policy, laws and other social issues, as implicated by the wide range of research subjects that was accorded to public health in our study.

\section{CONCLUSION}

In conclusion, the present analysis found that the proportion of the overall scientific literature that is ARD-related has declined substantially over the last quarter century, and that this decline was enhanced by a decrease in emphasis on public health, although with notable intercountry variations. The future direction of ARD-related research warrants a review in consideration of the ongoing situation on asbestos and foreseeable situation for ARD reflected in the pertinent WHO/ILO recommendations. Setting research priorities with improved emphasis on public health is essential to fill the gaps in research on ARD.

\section{Author affiliations}

${ }^{1}$ Department of Occupational Safety and Health, College of Public Health, China Medical University, Taichung, Taiwan

${ }^{2}$ Asbestos Diseases Research Institute, Concord Clinical School, University of Sydney, Rhodes, New South Wales, Australia

${ }^{3}$ Epidemiology and Biostatistics, Department of Environmental and Occupational Health, University of Nevada, School of Community Health Sciences, Las Vegas, Nevada, USA

${ }^{4}$ National Centre for Asbestos Related Diseases, University of Western Australia, Nedlands, Western Australia, Australia

${ }^{5}$ International Commission on Occupational Health, Milano, Italy

${ }^{6}$ Department of Environmental Health, Rollins School of Public Health, Emory University, Canton, Georgia, USA

${ }^{7}$ School of Public Health, University of Sydney, Sydney, New South Wales, Australia

Acknowledgements We thank the editors at English Manager Science Editing for their careful copyediting and Victoria Keena at the Asbestos Diseases Research Institute for her kind clerical assistance.
Contributors R-TL contributed to the study design, literature reviews, data collection, data analysis, data interpretation and writing of the manuscript. KT contributed to the study design, data classification, data interpretation and writing of the manuscript. MJS contributed to data classification and writing of the manuscript. L-CC contributed to statistical analysis, data interpretation and writing of the manuscript. SF, JT, RL and TD contributed to the data interpretation and writing of the manuscript.

Funding This work was supported by the Asbestos Safety and Eradication Agency (AA17/22) and the China Medical University (CMU106-N-27).

Competing interests RL testifies in asbestos litigation.

Patient consent Not required.

Provenance and peer review Not commissioned; externally peer reviewed.

Data sharing statement All available data can be obtained by contacting the corresponding author.

Open access This is an open access article distributed in accordance with the Creative Commons Attribution Non Commercial (CC BY-NC 4.0) license, which permits others to distribute, remix, adapt, build upon this work non-commercially, and license their derivative works on different terms, provided the original work is properly cited, appropriate credit is given, any changes made indicated, and the use is non-commercial. See: http:// creativecommons.org/licenses/by-nc/4.0/.

\section{REFERENCES}

1. World Health Organization. Elimination of asbestos-related diseases. Geneva, Switzerland: World Health Organization. 2006 http://apps.who.int/iris/bitstream/10665/69479/1/WHO_SDE_OEH_ 06.03_eng.pdf (accessed 26 Nov 2017).

2. World Health Organization. Chrysotile asbestos. Geneva, Switzerland: World Health Organization. 2014 http://www.who.int/ ipcs/assessment/public_health/asbestos/en/ (accessed 6 Jun 2017).

3. International Labour Organization. Resolution concerning asbestos. International Labour Organization. 2006 http://www.ilo.org/ safework/info/standards-and-instruments/WCMS_108556/lang-en/ index.htm (accessed 5 Dec 2017).

4. Kazan-Allen L. Chronology of national asbestos bans. 2017 http:// www.ibasecretariat.org/chron_ban_list.php

5. Marsili D, Terracini B, Santana VS, et al. Prevention of asbestosrelated disease in countries currently using asbestos. Int J Environ Res Public Health 2016;13:494.

6. USGS. United States Geological Survey. 2015 minerals yearbook: asbestos [advance release]. Reston, Virginia: USGS. 2016 https:// minerals.usgs.gov/minerals/pubs/commodity/asbestos/myb1-2015asbes.pdf (accessed 3 Nov 2017).

7. Institute for Health Metrics and Evaluation. GBD compare data visualization. Seattle, WA: IHME, University of Washington. 2016 http://vizhub.healthdata.org/gbd-compare (accessed 12 Oct 2017).

8. Odgerel CO, Takahashi K, Sorahan T, et al. Estimation of the global burden of mesothelioma deaths from incomplete national mortality data. Occup Environ Med 2017;74:851-8.

9. Takahashi K, Landrigan PJ. Collegium Ramazzini. The Global Health Dimensions of Asbestos and Asbestos-Related Diseases. Ann Glob Health 2016;82:209-13.

10. World Health Organization. The WHO strategy on research for health. France: World Health Organization. 2012 http://www.who.int/ phi/WHO_Strategy_on_research_for_health.pdf (accessed 20 Sep 2017).

11. Pang T, Sadana R, Hanney S, et al. Knowledge for better health: a conceptual framework and foundation for health research systems. Bull World Health Organ 2003;81:815-20.

12. Kuruvilla S, Mays N, Pleasant A, et al. Describing the impact of health research: a Research Impact Framework. BMC Health Serv Res 2006;6:18.

13. Durieux V, Gevenois PA. Bibliometric indicators: quality measurements of scientific publication. Radiology 2010;255:342-51.

14. Ugolini D, Bonassi S, Cristaudo A, et al. Temporal trend, geographic distribution, and publication quality in asbestos research. Environ $\mathrm{Sci}$ Pollut Res Int 2015;22:6957-67.

15. Ugolini D, Neri M, Casilli C, et al. A bibliometric analysis of scientific production in mesothelioma research. Lung Cancer 2010;70:129-35.

16. Clarivate Analytics. Searching the topic field. 2018 https://images. webofknowledge.com/images/help/WOK/hs_topic.html (accessed 8 May 2018). 
17. Clarivate Analytics. InCites. Report created: 19 October 2017. Data Source: Web of Science. 2017 https://incites.thomsonreuters.com/ (accessed 19 Oct 2017).

18. Clarivate Analytics. Web of Science research areas. $2017 \mathrm{http}: / /$ ipscience-help.thomsonreuters.com/inCites2Live/filterValuesGroup/ researchAreaSchema/wosDetail/wosCategories.html (accessed 1 May 2017).

19. Kim HJ, Fay MP, Feuer EJ, et al. Permutation tests for joinpoint regression with applications to cancer rates. Stat Med 2000;19:335-51.

20. Lin $X$, Zhang $D$. Inference in generalized additive mixed models by using smoothing splines. J R Statis Soc B 1999;61:391-400.

21. British Geological Survey. World mineral statistics. 2017 https:// www.bgs.ac.uk/mineralsuk/statistics/wms.cfc?method=searchWMS (accessed 1 Jul 2017).

22. UNESA. World population prospects, the 2015 revision. Department of economic and social affairs, United Nations. 2017 https://esa.un. org/unpd/wpp/Download/Standard/Population/ (accessed 17 May 2017)

23. Alpha NA. Significance level of test. California, United States: SAGE Publications, 2008.

24. International Labour Organization, World Health Organization. Outline for the development of national programmes for elimination of asbestos-related diseases. International Labour Organization and World Health Organization. 2007 http://www.who.int/occupational health/publications/elim_asbestos_doc_en.pdf (accessed 4 Oct 2017).

25. Smits PA, Denis JL. How research funding agencies support science integration into policy and practice: an international overview. Implement Sci 2014;9:12.
26. Conceição C, Leandro A, McCarthy M. National support to public health research: a survey of European ministries. BMC Public Health 2009;9:203.

27. McCarthy M, Conceição C, Grimaud O, et al. Competitive funding and structures for public health research in European countries. Eur J Public Health 2013;23(Suppl 2):39-42.

28. McKee M, Stuckler D, Basu S. Where there is no health research: what can be done to fill the global gaps in health research? PLoS Med 2012;9:e1001209.

29. Eurogip. Asbestos-related occupational diseases in Europe. Recognition, statistics, specific systems. Paris, France: Eurogip, 2006.

30. Banduch I, Lissner L. Asbestos-related occupational diseases in Central and East European countries. Hamburg, Germany: Kooperationsstelle Hamburg IFE, 2013.

31. García-Gómez M, Menéndez-Navarro A, López RC. Asbestosrelated occupational cancers compensated under the Spanish National Insurance System, 1978-2011. Int J Occup Environ Health 2015;21:31-9.

32. Cianciara D, Piotrowicz M, Gajewska M, et al. Is there the gap in public health literature in Europe? Ann Agric Environ Med 2013;20:631-6

33. Henderson DW, Rantanen J, Barnhart S, et al. Asbestos, asbestosis, and cancer: the Helsinki criteria for diagnosis and attribution. Scand $J$ Work Environ Health 1997;23:311-6.

34. Wolff H, Vehmas T, Oksa P, et al. Asbestos, asbestosis, and cancer, the Helsinki criteria for diagnosis and attribution 2014 recommendations. Scand J Work Environ Health 2015;41:5-15.

35. Marsili D, Angelini A, Bruno C, et al. Asbestos ban in Italy: a major milestone, not the final cut. Int J Environ Res Public Health 2017;14:1379. 